\title{
ANÁLISE DE CONFIABILIDADE ESTRUTURAL DE PROBLEMAS BASEADOS NA MECÂNICA DOS SÓLIDOS
}

\author{
Structural Reliability Analysis of problems based on Solid Mechanics \\ Vinícius Favaretto Defiltro', Wellison José de Santana Gomes ${ }^{2}$
}

Recebido em 26 de novembro de 2015; recebido para revisão em 08 de março de 2016; aceito em 08 de maio de 2016; disponível on-line em 04 de julho de 2016.

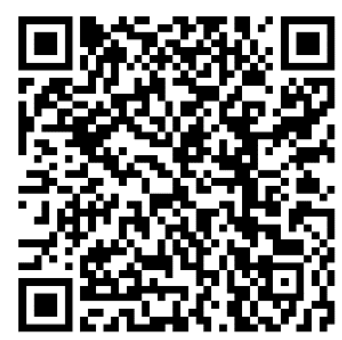

PALAVRAS CHAVE:

Mecânica dos sólidos;

Análise estrutural;

Confiabilidade estrutural;

Simulação de Monte

Carlo;

Probabilidade de falha.

\section{KEYWORDS:}

Solid mechanics;

Structural analysis;

Structural reliability;

Monte Carlo simulation;

Probability of faiture.

RESUMO: A Mecânica dos Sólidos, a partir de hipóteses simplificadoras, fornece modelos de cálculo que podem ser aplicados a vários problemas estruturais e estabelece as bases e o entendimento para o desenvolvimento de teorias e a construção de modelos mais complexos. Entretanto, dentro deste contexto, é comum desprezar as incertezas inerentes às propriedades dos materiais envolvidos, às condições de contorno e à geometria do problema. Neste artigo, ferramentas da Teoria da Confiabilidade Estrutural são aplicadas a problemas estruturais baseados na Mecânica dos Sólidos no intuito de analisá-los considerando algumas das incertezas envolvidas. Para isso, o método de Simulação de Monte Carlo é empregado na análise de confiabilidade de duas vigas. No estudo da primeira estrutura, busca-se investigar a influência da correlação entre variáveis aleatórias na probabilidade de falha do elemento estrutural. Na segunda estrutura, analisa-se o efeito da utilização de materiais com diferentes comportamentos (frágeis ou dúcteis) e, consequentemente, diferentes critérios de ruptura, sobre a probabilidade de falha estimada. Verifica-se que as análises de confiabilidade estrutural podem fornecer muitas informações que estão fora do escopo das soluções determinísticas. Tais informações permitem uma avaliação mais precisa da segurança estrutural e podem também levar a um melhor entendimento do modelo estrutural em questão.

ABSTRACT: The Solid Mechanics, from simplifying assumptions, provides calculation models that can be applied to various structural problems and establishes the foundation and the understanding for the development of theories and the construction of more complex models. However, within this context, it is common to despise the uncertainties inherent to the properties of the materials involved, the boundary conditions and the geometry of the problem. In this article, Structural Reliability Theory tools are applied to structural problems based on Solid Mechanics in order to analyze them considering some of the uncertainties involved. For this, the Monte Carlo simulation method is used in the reliability analysis of two beams. In the first structure, study seeks to investigate the influence of correlation between random variables on the probability of failure of the structural element. In the second one, the effect of using materials with different behavior (ductile or brittle) and, consequently, different rupture criteria on the estimated probability of failure is analyzed. Structural reliability analysis can provide information which usually is outside the scope of deterministic solutions. Such information enables a more accurate assessment of structural safety and may lead to a better understanding of the structural model in question.

\footnotetext{
* Contato com os autores:
}

${ }^{1}$ e-mail: vinicius.favaretto@grad.ufsc.br (V. F. Defiltro)

Graduando em Engenharia Civil pela Universidade Federal de Santa Catarina (UFSC).

2e-mail: wellison.gomes@ufsc.br (W. J. S. Gomes)

Eng. Civil, Doutor, Professor no departamento de Engenharia Civil pela Universidade Federal de Santa Catarina (UFSC). 


\section{INTRODUÇÃO}

A análise e projeto de estruturas requer a adoção de modelos estruturais que representem suficientemente bem o comportamento real da estrutura estudada; isto é, apesar das hipóteses e simplificações do modelo, a resposta do mesmo deve ser a mais próxima possível daquela apresentada pela estrutura real.

Tradicionalmente, os projetos se baseiam em métodos determinísticos ou semi-probabilísticos: a resistência de um elemento é determinada de forma que iguale o carregamento atuante ou o exceda com certa margem. O quociente entre a resistência e o carregamento (fator de segurança) é considerado uma medida da segurança da estrutura. Nas normas/códigos para projeto de estruturas os valores das cargas, resistências e fatores de segurança são prescritos (Sorensen, 2004), de maneira que as estruturas usuais satisfaçam a requisitos mínimos aceitáveis de segurança durante o período denominado vida útil. Entretanto, incertezas inevitavelmente estarão presentes na geometria, nos materiais e parâmetros de carga das estruturas, bem como no próprio modelo e no processo de análise (BECK e ROSA, 2006). Devido à não consideração direta destas incertezas, os métodos determinísticos e semi-probabilísticos não permitem quantificar o grau de segurança da estrutura, nem verificar a influência sobre a confiabilidade de parâmetros estruturais associados a resistências ou solicitações.

$\mathrm{Na}$ confiabilidade estrutural, é comum utilizar como medida de (in)segurança a chamada probabilidade de falha (PF), uma medida da propensão à falha por parte da estrutura (Beck, 2014), ou o índice de confiabilidade a ela associado. Assim, previsões de desempenho estrutural são efetuadas em um sentido probabilístico (HAUKAAS e DER KIUREGHIAN, 2004). Em Beck (2014), afirma-se ainda que ações e resistências não são necessariamente independentes (por exemplo, o peso próprio se relaciona diretamente com as dimensões da estrutura), indicando que um modelo representativo deve considerar tais relações.

Em virtude disso, neste artigo são aplicadas ferramentas da Teoria da Confiabilidade Estrutural na análise de estruturas cujas respostas são descritas por modelos baseados na Mecânica dos Sólidos. Tem-se como objetivo determinar probabilidades de falha, verificando a influência da correlação entre variáveis aleatórias que descrevem as incertezas dos problemas, bem como da adoção de diferentes critérios de falha, na PF estimada. Ressalta-se que os exemplos aqui apresentados comprovam a existência destas influências de uma forma não necessariamente inédita. A dependência entre correlação entre variáveis aleatórias e a probabilidade de falha, por exemplo, já foi citada em outras referências, tais como FRANGOPOL et al. (1996) e SOARES (2001).

\section{PRINCÍPIOS DA ANÁLISE DE CONFIABILIDADE}

A análise de confiabilidade estrutural tem como principal objetivo quantificar as possibilidades de um dado sistema estrutural não responder conforme desejado, devido às incertezas inerentes às suas propriedades físicas e geométricas e às condições de contorno a ele aplicadas.

No intuito de distinguir respostas estruturais desejadas das respostas não desejadas, são definidos os estados limites. Cada modo de falha (resposta indesejada) dá origem a um estado limite; estes, por sua vez, podem ser quantificados através das denominadas equações de estado limite. Sendo $\mathbf{X}=\left(X_{1}, X_{2}, \ldots, X_{n}\right)$ o vetor que contém as $n$ variáveis aleatórias que descrevem as incertezas do problema, a equação de estado limite $g$ é descrita como a Equação 1:

$$
g(\mathbf{X})=g\left(X_{1}, X_{2}, \ldots, X_{n}\right)=0 \quad \text { Eq.[1] }
$$

Cada função de estado limite pode ser interpretada em termos de resistência e solicitação, conforme a Equação 2:

$$
g(\mathbf{X})=R\left(X_{1}, \ldots, X_{k}\right)-S\left(X_{m}, \ldots, X_{n}\right) \quad \text { Eq.[2] }
$$

Em que:

$R=$ variável aleatória, função dos parâmetros de resistência;

$S$ = variável aleatória, função dos parâmetros de solicitação;

$k$ = quantidade de variáveis aleatórias que afetam a resistência;

$(n-m)=$ quantidade de variáveis aleatórias que afetam a solicitação. 
Assim, $g \leq 0$ representa a falha e $g>0$ representa sobrevivência da estrutura. Essa definição permite dividir o domínio, $\Omega$, da função $g(\mathbf{x})$ em dois subconjuntos, considerando como argumento da função vetores de realizações das variáveis aleatórias, $\mathbf{x}$ :

- Domínio de Falha:

$$
\Omega_{f}=\{\mathbf{x} \mid g(\mathbf{x}) \leq 0\}
$$

- Domínio de Segurança ou Sobrevivência: $\Omega_{s}=\{\mathbf{x} \mid g(\mathbf{x})>0\}$

Considerando a função conjunta de densidade de probabilidade das variáveis aleatórias contidas em $\mathbf{X}, f_{\mathrm{x}}(\mathbf{x})$, a probabilidade de falha é definida pela seguinte integral (Equação 3):

$$
P F=\int_{\Omega_{f}} f_{\mathbf{x}}(\mathbf{x}) d \mathbf{x}
$$

A integral sobre o domínio de falha pode ser substituída por uma integral sobre todo o domínio adotando-se uma função indicadora $I(\mathbf{x})$, que assume valor unitário caso $\mathbf{x}$ pertença ao domínio de falha e zero caso contrário. A integral passa a ser dada pela Equação 4:

$$
P F=\int_{\Omega} I(\mathbf{x}) f_{\mathbf{X}}(\mathbf{x}) d \mathbf{x}
$$

Tanto a função $f_{\mathrm{x}}(\mathbf{x})$ quanto o domínio de falha são geralmente difíceis de determinar e na maioria dos problemas estruturais não há expressões analíticas que os definam. Isto ocorre devido a fatores tais como falta de informação a respeito da variação conjunta das variáveis aleatórias, diferenças entre distribuições de probabilidades que descrevem cada uma das variáveis aleatórias e existência de relações não lineares na equação de estado limite.

Os métodos que têm sido utilizados no campo da confiabilidade estrutural para computar ou estimar esta integral são divididos em dois grupos: os métodos aproximados, tais como o método de confiabilidade de primeira ordem (FORM - First Order Reliability Method), que em geral trabalham com aproximações da função de estado limite $\mathbf{g}(\mathbf{X})$; e os métodos exatos, que tentam determinar a solução da integral diretamente.
Dentre os métodos exatos que podem ser empregados, estão a integração numérica e a simulação de Monte Carlo. Entretanto, a estratégia de integração numérica raramente é utilizada, devido à dificuldade de se computar $f_{\mathrm{x}}(\mathbf{x})$ para os pontos específicos de integração e à necessidade usual de muitos pontos de integração, associada ao grande número de variáveis aleatórias (alta dimensionalidade estocástica) comumente encontrado em problemas estruturais. O método de simulação de Monte Carlo (SMC), adotado neste artigo, é provavelmente o mais utilizado dentro da confiabilidade estrutural, devido à sua simplicidade, generalidade e robustez (as características deste método são mais profundamente discutidas em referências básicas de confiabilidade estrutural, e.g., ANG e TANG, 2007 e MELCHERS, 1999).

O método de Monte Carlo, em sua versão mais simples, consiste em gerar $n$ realizações das variáveis aleatórias contidas em $\mathbf{X}$ (pontos amostrais $\mathbf{x}_{\mathrm{i}}$, com $i=1,2, \ldots, n$ ) de acordo com a função conjunta de densidade de probabilidades, $f_{\mathrm{x}}(\mathbf{x})$, e estimar a probabilidade de falha por meio da Equação 5:

$$
P F=\frac{1}{N} \sum_{i=1}^{n} I\left(\mathbf{x}_{i}\right)
$$

Nota-se que a função indicadora, por depender de variáveis aleatórias, é também uma variável aleatória, e que a PF estimada pela Equação 5 corresponde à média da função indicadora avaliada nos pontos amostrais $\mathbf{x}_{\mathrm{i}}$. Por outro lado, como o estimador apresentado na Equação 5 depende de uma amostra aleatória, ele apresenta uma variância, que está associada ao erro estatístico da estimação, e pode ser visto também como uma variável aleatória.

A amostra necessária para estimar a probabilidade de falha pode ser gerada a partir das distribuições de probabilidade marginais das variáveis aleatórias envolvidas e da correlação entre as mesmas, que na prática são as informações geralmente disponíveis. Detalhes a respeito do procedimento de geração de amostras correlacionadas ou não-correlacionadas podem ser encontrados em várias referências da literatura 
(e.g., HAUKAAS e DER KIUREGHIAN, 2004; SORENSEN, 2004).

A precisão das estimativas obtidas pela Equação 5 depende diretamente do número de simulações realizadas e do valor da quantidade de interesse. Quanto maior o número de simulações, maior a precisão; quanto menor o valor da probabilidade de falha, maior o número de simulações necessário. Assim, para valores muito pequenos da probabilidade de falha, estimativas suficientemente precisas podem apresentar custos computacionais muito elevados ou até mesmo proibitivos.

Segundo Ditlevsen (2007), é extremamente difícil determinar, por meios teóricos, um tamanho amostral suficiente para se obter um resultado confiável. Uma das razões para tal é que a distribuição de probabilidades da função de estado limite, que depende de variáveis aleatórias e é também uma variável aleatória, é geralmente desconhecida. Entretanto, o que pode ser definido como suficiente depende do tipo de distribuição da(s) variável(eis) envolvidas, assim como do tempo de análise disponível (que por sua vez está atrelado à capacidade de processamento da máquina utilizada e eficiência do algoritmo desenvolvido) e ainda de outros fatores. $\mathrm{Na}$ literatura, enquanto em alguns casos são apresentadas expressões que permitem estimar um número aproximado de simulações necessárias de acordo com o coeficiente de variação requerido para a probabilidade de falha (Beck, 2014), em outras publicações opta-se for efetuar uma análise gráfica de convergência (GOMES et al., 2013; GOMES e BECK, 2014).

No presente artigo, optou-se por determinar o tamanho da amostra utilizando a segunda opção. Assim, em todos os problemas resolvidos, são efetuadas análises de convergência da PF estimada para determinar tamanhos de amostra que levem a resultados suficientemente precisos com esforços computacionais aceitáveis.

Apesar de existirem versões mais eficientes do método de Monte Carlo disponíveis na literatura (diferentes versões da SMC podem ser consultadas em Sorensen (2004) e Haukaas e Der Kiureghian (2004)), no presente artigo optou-se por utilizar a versão simples, devido à facilidade de implementação e ao fato da convergência desta versão não depender da escolha de parâmetros. Assim, para evitar custos computacionais proibitivos, são analisados problemas com valores de PF maiores do que os usualmente encontrados em análises de confiabilidade estrutural. Porém, esta escolha está de acordo com a definição de falha como sendo qualquer resposta além do regime elástico-linear, que por sua vez está vinculada à representação do comportamento estrutural por modelos simplificados da Mecânica dos Sólidos. Probabilidades de respostas além do regime elástico-linear são bem maiores do que probabilidades de falha reais das estruturas. Neste sentido, ressalta-se que o objetivo deste artigo é discutir algumas das vantagens de se aplicar análises de confiabilidade, ao invés de análises puramente determinísticas, mesmo quando o modelo estrutural em questão é relativamente simples. Assim, a escolha dos parâmetros aqui utilizados não segue valores reais e/ou obtidos experimentalmente, mas se justifica pelo objetivo de elevar a PF obtida, tanto como forma de diminuir o custo computacional como para evidenciar as conclusões formuladas, de forma didática.

\section{INFLUÊNCIA DA INSERÇÃO DE CORRELAÇÃO ENTRE VARIÁVEIS ALEATÓRIAS NA PROBABILIDADE DE FALHA}

Com o objetivo de estudar a influência sobre a PF da inserção de correlações entre variáveis aleatórias, considera-se a estrutura ilustrada na Figura 1.

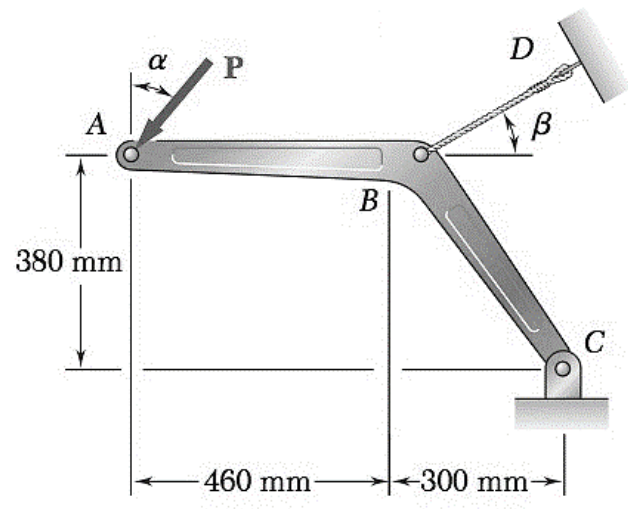

FIGURA 1: Geometria da primeira estrutura. FONTE: adaptado de Beer et al. (2014). 
Por meio de uma análise de equilíbrio estático na configuração inicial, obtêm-se a seguinte função de estado limite, expressa na Equação 6:

$$
g(R, P, \alpha, \beta)=R-\frac{(19 \times \sin \alpha+38 \times \cos \alpha)}{(15 \times \sin \beta+19 \times \cos \beta)} \times P \quad \text { Eq. [6] }
$$

Em que:

$R=$ representa a força de tração (resistência) na corda;

$P=$ representa o valor da carga concentrada atuante;

$g$ = função de estado limite da estrutura.

Verifica-se que os parâmetros $\alpha$ e $\beta$ podem mudar significativamente a função de estado limite, uma vez que os cossenos e senos destes ângulos são multiplicados por fatores bem maiores do que 1 na função. Neste sentido, inicialmente determina-se o tamanho da amostra a ser utilizado considerando $R$ e $P$ variáveis aleatórias com tipos de distribuição e parâmetros indicados na Tabela 1 , e os parâmetros $\alpha$ e $\beta$ com valores determinísticos apresentados na Tabela 2. Em seguida, determinam-se os valores de $\alpha$ e $\beta$ que conduzem às situações extremas de menor e maior probabilidade de falha, respectivamente. 0 problema final a ser analisado tem como variáveis aleatórias não apenas $R$ e $P$, conforme descrito na Tabela 1, mas também os parâmetros $\alpha$ e $\beta$, com médias iguais aos valores relacionados às situações extremas identificadas.

Para a configuração específica do problema descrita pelas Tabelas 1 e 2, o gráfico de convergência ilustrado na Figura 2 é obtido. Nota-se que, para este caso, a função de estado limite se torna linear nas variáveis aleatórias (VA), o que permite calcular a PF exata para o problema, conforme apresentado em (BECK, 2014). De posse da PF exata, é possível não apenas analisar a convergência da PF estimada, mas também validar o código computacional que foi implementado. Levando em conta o gráfico de convergência obtido, adota-se um tamanho amostral de $5 \times 10^{6}$ para as análises subsequentes.

TABELA 1: Variáveis aleatórias utilizadas na determinação do tamanho da amostra.

\begin{tabular}{cccc} 
Variável & Tipo de Distribuição & Média (KN) & Desvio Padrão (KN) \\
\hline$R$ & Normal & 110 & 10 \\
\hline$P$ & Normal & 60 & 15 \\
\hline & & & FONTE: Autoria própria.
\end{tabular}

TABELA 2: Parâmetros utilizados na determinação do tamanho da amostra.

\begin{tabular}{cc} 
Variável & Valor (graus) \\
\hline$\alpha$ & 38 \\
\hline$\beta$ & 0 \\
\hline
\end{tabular}

FONTE: Autoria própria.

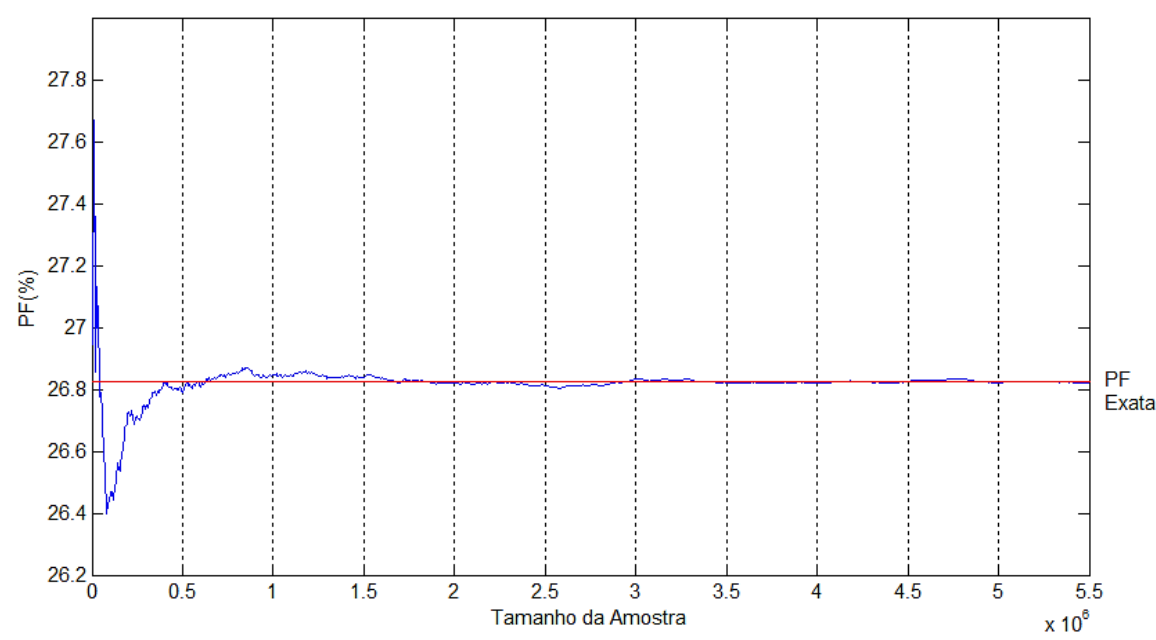

FIGURA 2: Convergência da PF com o aumento do tamanho da amostra. FONTE: Autoria própria. 
As combinações dos ângulos $\alpha$ e $\beta$ que levam às situações extremas são determinadas variando estes ângulos dentro do intervalo de 0 a 45 graus e computando a probabilidade de falha para as várias combinações possíveis, como ilustrado na Figura 3 . Os pares de valores associados 2
Os pares de valores associados a cada uma das situações são denominados "geometria pior" e "geometria melhor" e apresentados na Tabela 3.

De posse dos valores relacionados às duas situações extremas, são definidos os parâmetros das distribuições de probabilidade a serem considerados, conforme apresentado na Tabela 4.

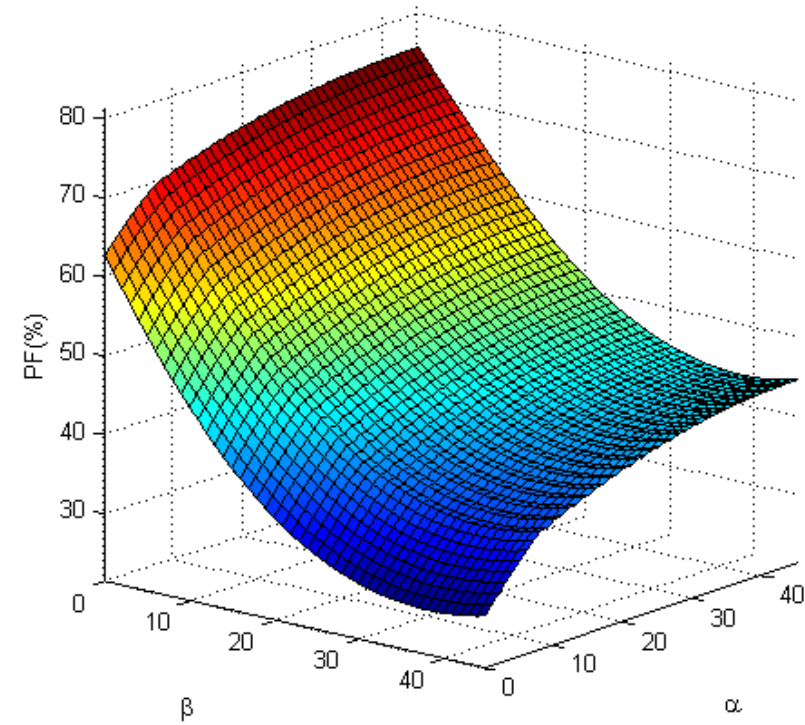

FIGURA 3: PF para cada combinação de $\alpha$ e $\beta$. FONTE: Autoria própria.

TABELA 3: Valores de $\alpha$ e $\beta$ para as geometrias "Melhor" e "Pior"

Geometria Melhor

$\begin{array}{cc}\alpha=0 & \mathrm{PF}=26,8648 \%\end{array}$

\section{Geometria Pior}

\begin{tabular}{cc}
$\alpha=38$ & $\mathrm{PF}=75,3125 \%$ \\
\hline$\beta=0$ &
\end{tabular}

FONTE: Autoria própria.

TABELA 4: Variáveis aleatórias utilizadas no cálculo da probabilidade de falha.

\begin{tabular}{ccccc} 
Variável & Tipo de Distribuição & Unidade & Média & Desvio Padrão \\
\hline$R$ & Normal & $\mathrm{kN}$ & 110 & 10 \\
\hline$P$ & Normal & $\mathrm{kN}$ & 60 & 15 \\
\hline$\alpha_{\text {melhor }}$ & Normal & graus & 0 & 3 \\
\hline$\beta_{\text {melhor }}$ & Normal & graus & 26,5 & 3 \\
\hline$\alpha_{\text {pior }}$ & Normal & graus & 38 & 3 \\
\hline$\beta_{\text {pior }}$ & Normal & graus & 0 & 3 \\
\hline & & & & FONTE: Autoria própria.
\end{tabular}

FONTE: Autoria própria. 


\subsection{INSERÇÃO DE CORRELAÇÕES}

Usualmente em confiabilidade estrutural, quando se trabalha com variáveis correlacionadas, é utilizado um coeficiente de correlação $c$ (sendo que $-1 \leq c \leq 1)$ para cada par de variáveis. 0 coeficiente $c$ é uma medida da relação linear entre o par de variáveis aleatórias, variando de $c=-1$, que indica uma correlação linear negativa perfeita, a $c=1$, que indica uma correlação linear positiva perfeita. Estes coeficientes são armazenados em uma matriz $\mathrm{R}=\left[c_{i j}\right]$, cujos índices representam as variáveis aleatórias da estrutura e a diagonal contém valores unitários (HAUKAAS e DER KIUREGHIAN, 2004). No presente artigo os valores $c_{i j}$ foram escolhidos arbitrariamente, para estudar os efeitos de diferentes correlações. Na prática, tais

As características da matriz de correlações, assim como aspectos teóricos e demonstrações para implementação de geradores de números aleatórios com correlação podem ser encontrados, por exemplo, em (HAUKAAS e DER KIUREGHIAN, 2004).

Para cada uma das geometrias são considerados três casos distintos, descritos na Tabela 5:

\subsection{RESULTADOS E ANÁLISES}

Os resultados obtidos para o caso I estão sintetizados na Tabela 6.

Percebe-se que a correlação entre as variáveis representativas da geometria do problema não afeta significativamente a PF. Isto significa que, neste caso, os efeitos produzidos pela correlação nos termos relacionados aos ângulos $\alpha$ e $\beta$ praticamente se anulam. Ou seja, a inserção de correlação desloca conteúdo de probabilidades de regiões de falha, dentro do espaço estocástico definido por $\alpha$ e $\beta$, para outras regiões de falha, e não para regiões de sobrevivência. Para o caso II os seguintes resultados são obtidos conforme a Tabela 7.

Neste caso ocorre o oposto; a inserção de correlação na geometria melhor desloca conteúdo de probabilidades de regiões de falha, dentro do espaço estocástico definido por $\alpha$ e $\beta$, para regiões de sobrevivência (o oposto é observado para a geometria pior); alterando significativamente a PF. Assim, a consideração das variáveis correlacionadas diminui ainda mais a PF da melhor geometria e aumenta a PF da pior geometria.

Para o terceiro e último caso analisado, os resultados são apresentados na Tabela 8.

Neste último caso, as variações da PF também são significativas, o que confirma as observações feitas anteriormente.

$\mathrm{Na}$ tentativa de explicar o efeito das correlações na resposta da estrutura (PF) e levantar conclusões que se apliquem a casos gerais, verificou-se que uma análise mais aprofundada do assunto é necessária. Por estar além do escopo deste trabalho, optou-se por deixar esta análise para trabalhos futuros.

TABELA 5: Casos de estudo para cada geometria ("melhor" e "pior").

\begin{tabular}{cl} 
Caso & \multicolumn{1}{c}{ Descrição } \\
\hline । & $\begin{array}{l}\text { Aumento gradativo do coeficiente de correlação entre as } \\
\text { variáveis } \alpha \text { e } \beta \text {. Não há correlação entre } R \text { e } P .\end{array}$ \\
\hline II & $\begin{array}{l}\text { Aumento gradativo do coeficiente de correlação entre as } \\
\text { variáveis } R \text { e } P \text {. Não há correlação entre } \alpha \text { e } \beta .\end{array}$ \\
\hline III & $\begin{array}{l}\text { Aumento gradativo do coeficiente de correlação tanto entre as } \\
\text { variáveis } R \text { e } P \text { quanto entre as variáveis } \alpha \text { e } \beta .\end{array}$
\end{tabular}

FONTE: Autoria própria. 
TABELA 6: Probabilidade de falha estimada para diferentes coeficiente de correlação entre $\alpha$ e $\beta$.

Geometria Pior

\begin{tabular}{ccc}
\hline Coef. Corr. entre $\boldsymbol{\alpha}$ e $\boldsymbol{B}$ & Coef. Corr. entre $\boldsymbol{R}$ e $\boldsymbol{P}$ & PF (\%) \\
\hline 0,3 & 0 & 75,3471 \\
\hline 0,5 & 0 & 75,3312 \\
\hline 0,9 & 0 & 75,3422 \\
\hline Coef. Corr. entre $\boldsymbol{\alpha}$ e $\boldsymbol{B}$ & Geometria Melhor & PF (\%) \\
\hline 0,3 & Coef. Corr. entre $\boldsymbol{R}$ e $\boldsymbol{P}$ & 26,8822 \\
\hline 0,5 & 0 & 26,8560 \\
\hline 0,9 & 0 & 26,8990 \\
\hline
\end{tabular}

TABELA 7: Probabilidade de falha estimada para diferentes coeficiente de correlação entre $R$ e $P$.

Geometria Pior

Geometria Melhor

\begin{tabular}{cccccc}
\hline Coef. Corr. Entre $\boldsymbol{\alpha}$ e $\boldsymbol{B}$ & $\begin{array}{c}\text { Coef. Corr. } \\
\text { Entre } \boldsymbol{R} \text { e } \boldsymbol{P}\end{array}$ & PF (\%) & $\begin{array}{c}\text { Coef. Corr. } \\
\text { Entre } \boldsymbol{\alpha} \text { e } \boldsymbol{B}\end{array}$ & $\begin{array}{c}\text { Coef. Corr. } \\
\text { Entre } \boldsymbol{R} \text { e } \boldsymbol{P}\end{array}$ & PF (\%) \\
\hline 0 & 0,3 & 77,2667 & 0 & 0,3 & 24,3628 \\
\hline 0 & 0,5 & 78,8449 & 0 & 0,5 & 22,0944 \\
\hline 0 & 0,9 & 82,9868 & 0 & 0,9 & 15,2216 \\
\hline 0 & $-0,9$ & 71,2979 & 0 & $-0,9$ & 31,5134 \\
\hline
\end{tabular}

TABELA 8: Probabilidade de falha estimada para diferentes coeficiente de correlação entre $\alpha$ e $\beta$ e entre $R$ e $P$.

Geometria Pior

Geometria Melhor

\begin{tabular}{cccccc}
\hline $\begin{array}{c}\text { Coef. Corr. entre } \boldsymbol{\alpha} \\
\text { e } \boldsymbol{B}\end{array}$ & $\begin{array}{c}\text { Coef. Corr. } \\
\text { entre } \boldsymbol{R} \text { e } \boldsymbol{P}\end{array}$ & PF (\%) & $\begin{array}{c}\text { Coef. Corr. } \\
\text { entre } \boldsymbol{\alpha} \text { e } \boldsymbol{~}\end{array}$ & $\begin{array}{c}\text { Coef. Corr. } \\
\text { entre } \boldsymbol{R} \text { e } \boldsymbol{P}\end{array}$ & PF (\%) \\
\hline 0,3 & 0,3 & 77,2554 & 0,3 & 0,3 & 24,3066 \\
\hline 0,5 & 0,5 & 78,8196 & 0,5 & 0,5 & 22,1154 \\
\hline 0,9 & 0,9 & 82,9930 & 0,9 & 0,9 & 15,2249 \\
\hline
\end{tabular}

FONTE: Autoria própria.

\section{DETERMINAÇÃO DA PROBABILIDADE DE FALHA DE UMA VIGA BI-APOIADA PARA DIFERENTES CRITÉRIOS DE FALHA}

O comportamento de uma estrutura qualquer depende diretamente do material que a constitui. Diferentes materiais apresentam diferentes propriedades mecânicas e podem não romper da mesma forma ou pelo mesmo motivo.
Assim, cada tipo de material demanda um modelo estrutural que considere um critério de falha apropriado, entendendo-se por critério de falha aquele que prevê a ocorrência ou não de ruptura de um material quando o mesmo é submetido a um estado de tensão qualquer. Mais informações acerca das propriedades dos materiais e critérios de falha podem ser obtidas, por exemplo, em (BEER et al., 2014). 
Nesta seção estuda-se a influência da utilização de diferentes materiais (e, portanto, diferentes critérios de falha) na PF da estrutura. A estrutura analisada, ilustrada na Figura 4, é uma viga bi-apoiada e isostática, com duas cargas concentradas e uma distribuída e com seção transversal retangular e simétrica com relação à vertical. A geometria do problema é considerada a mesma para todos os casos estudados.

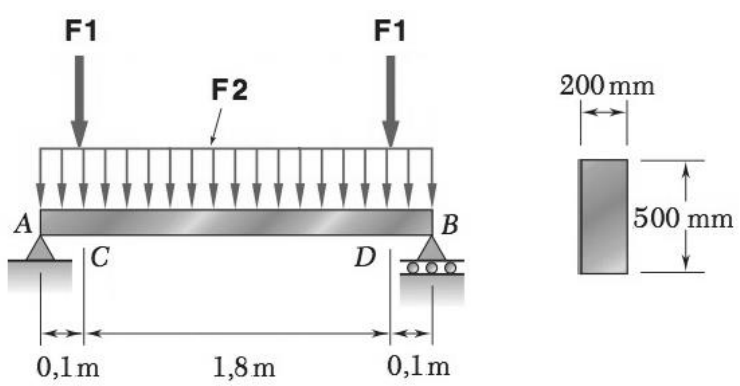

FIGURA 4: Geometria da segunda estrutura. FONTE: Adaptado de Beer et al. (2014).
Efetua-se uma análise plana de tensões para dois tipos de material distintos: um frágil e outro dúctil, mas com propriedades mecânicas semelhantes. A probabilidade de falha (probabilidade de resposta além do regime elástico) é calculada considerando: os dois tipos de material; três diferentes carregamentos, aleatórios e com distribuição Normal, com parâmetros descritos na Tabela 9; e três diferentes razões entre as tensões admissíveis ( $\sigma$ e $\tau$ ), determinísticas, conforme Tabela 10.

Neste problema, a falha da estrutura ocorre quando a tensão atuante na viga supera a tensão admissível e esta verificação é feita de acordo com o critério de falha adotado.

De forma geral, a falha pode ser descrita pela equação 7 :

$g(\sigma, \tau)=R\left(\sigma_{\text {adm }}, \tau_{\text {adm }}\right)-S\left(\sigma_{\text {atuante }}, \tau_{\text {atuante }}\right) \quad$ Eq.[7]

\begin{tabular}{cccccc}
\multicolumn{7}{c}{ TABELA 9: Casos de estudo com diferentes carregamentos. } \\
\hline \multirow{2}{*}{ Caso } & Variável & Tipo & Un. & $\boldsymbol{\mu}$ & $\boldsymbol{\sigma}$ \\
\hline & $F 1$ & Concentrada & $\mathrm{kN}$ & 100 & 10 \\
\hline \multirow{2}{*}{ II } & $F 2$ & Uniform. Dist. & $\mathrm{kN} / \mathrm{m}$ & 25 & 3 \\
\cline { 2 - 6 } & $F 1$ & Concentrada & $\mathrm{kN}$ & 100 & 10 \\
\hline III & $F 2$ & Uniform. Dist. & $\mathrm{kN} / \mathrm{m}$ & 20 & 3 \\
\cline { 2 - 6 } & $F 1$ & Concentrada & $\mathrm{kN}$ & 120 & 10 \\
\hline
\end{tabular}

FONTE: Autoria própria.

\begin{tabular}{cccc}
\hline & \multicolumn{3}{c}{ TABELA 10: Casos de estudo com diferentes tensões admissíveis. } \\
Caso & Parâmetro & Un. & Valor \\
\hline A & $\sigma_{a d m}$ & $\mathrm{MPa}$ & 3,2 \\
\cline { 2 - 3 } & $\tau_{a d m}$ & $\mathrm{MPa}$ & 1,3 \\
\hline $\mathrm{B}$ & $\sigma_{a d m}$ & $\mathrm{MPa}$ & 2,8 \\
\hline & $\tau_{a d m}$ & $\mathrm{MPa}$ & 1,4 \\
\hline $\mathrm{C}$ & $\sigma_{a d m}$ & $\mathrm{MPa}$ & 2,8 \\
\hline & $\tau_{a d m}$ & $\mathrm{MPa}$ & 1,8 \\
\hline
\end{tabular}




\section{Em que:}

$g$ = função de estado limite da estrutura;

$R=$ variável aleatória, função das tensões admissíveis;

$S=$ variável aleatória, função combinação das tensões atuantes.

O critério utilizado para descrever a falha do material dúctil foi o Critério de Tresca ou critério da força cisalhante máxima, enquanto que para o material frágil foi utilizada a Teoria de Rankine ou teoria da máxima tensão normal. Detalhes sobre estes critérios e suas propriedades podem ser encontrados em (BEER et al., 2014).

Além dos carregamentos, os módulos de elasticidade longitudinal, $E$, e transversal, $G$, e as dimensões da seção transversal, base $b$ e altura $h$, são consideradas variáveis aleatórias, conforme descrição apresentada na Tabela 11.

\subsection{RESULTADOS E ANÁLISES}

Os resultados obtidos em termos de probabilidade de falha para todas as combinações de tensões admissíveis e de casos de carregamento são apresentados nas Tabelas 12 e 13, para os casos de material dúctil e frágil, respectivamente.

Ao se comparar os resultados das duas Tabelas, nota-se que as diferenças entre os valores da PF da estrutura são bastante significativas para vários casos analisados. Entretanto, para o caso B, no qual, os resultados são semelhantes para materiais frágeis e dúcteis.

Isto ocorre pois, para estes valores relativos das tensões admissíveis, a máxima tensão cisalhante e a máxima tensão normal atuantes na estrutura ocorrem no mesmo ponto, independentemente da tensão solicitante predominante, o que faz com que os dois critérios de falha conduzam aos mesmos resultados.

Porém, na prática, materiais dúcteis e materiais frágeis apresentam diferentes relações entre e, de maneira que as probabilidades de falha dificilmente seriam iguais, mesmo que sejam mantidas as condições de carregamento, geometria e vinculação.

Seguindo este raciocínio e analisando os demais resultados obtidos, verifica-se que a razão representa um parâmetro que caracteriza o desempenho de cada tipo de material. Para o problema estrutural e hipóteses consideradas:

O material dúctil apresenta melhor desempenho quando;

O material frágil apresenta melhor desempenho quando.

Ressalta-se que esta definição de desempenho não leva em conta as grandes capacidades de deformação associadas a materiais dúcteis e as implicações destas, por exemplo, na redistribuição de esforços.

TABELA 11: Valores utilizados para cada variável aleatória no cálculo da probabilidade de falha.

\begin{tabular}{cccccc} 
Variável & $\begin{array}{c}\text { Tipo de } \\
\text { Distribuição }\end{array}$ & Tipo & Unidade & Média & Desvio Padrão \\
\hline$E$ & Normal & Cte. Elástica & GPa & 50 & 1 \\
\hline$G$ & Normal & Cte. Elástica & GPa & 26 & 1 \\
\hline$b$ & Normal & Dimensão da Seção & $\mathrm{cm}$ & 20 & 2 \\
\hline$h$ & Normal & Dimensão da Seção & $\mathrm{cm}$ & 50 & 2 \\
\hline
\end{tabular}

FONTE: Autoria própria. 
TABELA 12: PF estimada (\%) para cada um dos casos considerando material dúctil.

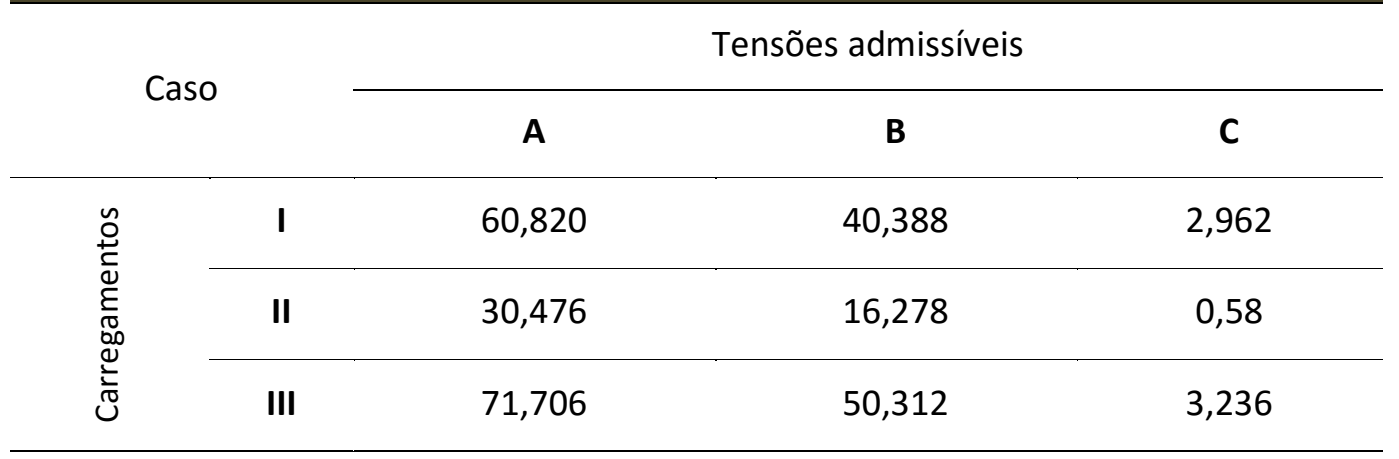

FONTE: Autoria própria.

\section{TABELA 13: PF estimada (\%) para cada um dos casos considerando material frágil.}

Tensões admissíveis

Caso

\begin{tabular}{ccc}
\hline A & B & C \\
13,278 & 40,848 & 40,818 \\
\hline 3,524 & 16,234 & 16,336 \\
\hline 15,636 & 50,248 & 50,174 \\
\hline
\end{tabular}

Os resultados obtidos chamam a atenção para o risco de se usar um critério de ruptura inadequado (que não descreva satisfatoriamente a forma de ruptura do material utilizado), o que pode levar a considerar uma estrutura em risco como sendo segura.

\section{CONCLUSÃO}

Neste artigo, através da utilização de ferramentas da confiabilidade estrutural e da Mecânica dos Sólidos, estudou-se o efeito de diversos parâmetros sobre a segurança das estruturas. Os resultados levaram às seguintes conclusões:

- Diferentes valores de correlação entre duas ou mais variáveis do problema podem levar a variações significativas no valor da probabilidade de falha. A inserção de correlações não pode ser assumida como sempre a favor ou contra a segurança, tal análise deve ser feita caso a caso. Portanto, é necessário caracterizar da melhor maneira possível as relações entre as variáveis aleatórias envolvidas em cada problema de confiabilidade, para que as respostas sejam condizentes com a realidade;

- A adoção de materiais com características frágeis ou dúcteis, mesmo que com propriedades mecânicas semelhantes, também pode levar a diferenças significativas no valor da probabilidade de falha. Isto se relaciona ao fato que diferentes critérios de ruptura podem levar a equações de estado limite significativamente diferentes, e indica a necessidade de utilização de critérios de ruptura adequados em análises de confiabilidade. Para o problema estudado (na seção 5), foi possível, inclusive, determinar valores da relação $\tau_{a d m} / \sigma_{a d m}$ (tensão cisalhante admissível sobre tensão axial admissível) a partir dos quais o 
desempenho de um material frágil seria melhor do que o desempenho de um material dúctil, desprezando, entretanto, a grande capacidade de deformação dos materiais dúcteis. Entretanto, ressalta-se que o valor limite encontrado diz respeito ao caso específico estudado, e que mais estudos são necessários para que esta constatação possa ser estendida a casos mais gerais.

As conclusões apresentadas mostram que, mesmo para problemas estruturais relativamente simples, a Teoria da Confiabilidade pode ser utilizada para obter informações sobre a resposta estrutural que não são fornecidas pelas abordagens mais comuns, sejam elas determinísticas ou semiprobabilísticas. Neste sentido, mesmo o método de simulação de Monte Carlo, que é um método bastante simples de implementar e aplicar, pode ser empregado. Porém, a simplicidade de aplicação e generalidade do método devem ser pesadas quanto ao esforço computacional requerido, que pode ser tornar proibitivo mesmo para problemas relativamente simples.

\section{AGRADECIMENTOS}

Os autores agradecem ao CNPq (Conselho Nacional de Desenvolvimento Científico e Tecnológico) pelo apoio financeiro sob a forma de bolsa do Programa Institucional de Bolsas de Iniciação Científica (PIBIC), concedida ao graduando.

\section{REFERÊNCIAS BIBLIOGRÁFICAS}

ANG, A. H. S.; TANG, W. H. Probability concepts in engineering: emphasis on applications to civil and environmental engineering. 2a edição, Nova lorque, NY, John Wiley and Sons., 2007.

BECK, A. T. Curso de Confiabilidade Estrutural. Universidade de São Paulo, Escola de Engenharia de São Carlos. São Carlos, SP, 2014, 236p.

BECK, A. T.; ROSA, E. Structural Reliability analysis using deterministic finite element programs. Latin America Journal of Solids and Structures, Vol 3, 2006, 197-222 p.
BEER, F. P.; JOHNSTON JR, E. R.; DEWOLF, J. T.; MAZUREK, D. F. Mecânica dos Materiais; McGraw Hill - Higher Education, 7ạ edição. Nova lorque, NY, 2014, 816p.

DITLEVSEN, O.; MADSEN, H. O. Structural Reliability Methods. Technical University of Denmark, Department of Mechanical Engineering. 2007, 361 p.

FRANGOPOL, D.M.; COROTIS, R. B.; RACKWITZ, R. Reliability and Optimization of Structural Systems. Elsevier, 1996.

GOMES, W. J. S.; BECK, A. T.; HAUKAAS, T. Optimal inspection planning for onshore pipelines subject to external corrosion. Reliability Engineering \& Systems Safety, v. 118, 2013, p. 18-27.

GOMES, W. J. S.; BECK, A. T. Optimal inspection planning and repair under random crack propagation. Engineering Structures, v. 69, p. 285-296, 2014.

MELCHERS, R. E. Structural reliability analysis and prediction. 2ª edição, Nova lorque, NY, John Wiley \& Son, 1999.

HAUKAAS, T.; DER KIUREGHIAN, A. Finite Element Reliability and Sensitivity Methods for PerformanceBased Earthquake Engineering. University of California, College of Engineering, Pacific Earthquake Engineering Research Center, PEER Report 2003/14. Berkeley, CA, 2004, 258 p.

SOARES, R. C. Um estudo sobre modelos mecânicoprobabilísticos para pórticos de concreto armado. Tese de Doutorado. Universidade de São Paulo, Escola de Engenharia de São Carlos. São Carlos, SP, 2001.

SORENSEN, J. D. Notes in Strutural Reliability Theory and Risk Analysis. Aalborg University, Institute of Building Technology and Structural Engineering. Aalborg, DEN, 2004, 230 p. 